\title{
Theoretical study for Laser Lines in Carbon like Zn (XXV)
}

\author{
Nahed Hosny Wahba*,1, Wessameldin Salah Abdelaziz ${ }^{1}$, Tharwat Mahmoud Alshirbeni ${ }^{2}$ \\ ${ }^{1}$ Department of Laser Applications in Metrology, Photochemistry, and Agriculture, National Institute of Laser Enhanced Sciences, \\ Cairo University, Giza, 12613, Egypt \\ ${ }^{2}$ Physics Department, Faculty of Science, Cairo University, Giza 12613, Egypt
}

\begin{tabular}{l} 
A R T I C L I N F O \\
\hline Article history: \\
Received: 06 July, 2021 \\
Accepted: 08 August, 2021 \\
Online: 16 August, 2021 \\
\hline Keywords: \\
FAC \\
C-like Zn (XXV) \\
Coupled Rate Equation \\
Reduced Population \\
Radiative Decay \\
Doppler Broadening Equation \\
Gain Coefficient
\end{tabular}

\section{Introduction}

X-ray lasers are a class of lasers in which gain has been demonstrated over various discrete wavelengths ranging from $3.56 \mathrm{~nm}$ to $46.9 \mathrm{~nm}$. Because of the very short-duration and highenergy excitation pulses required to generate these lasers [1], [2], photo excitation method [3], Electron collisional pumping method (ECP), charge transfer technique, electron collisional recombination process and dielectronic recombination pumping are examples of X-ray pumping procedures which using picoseconds chirped pulse amplification (CPA) pulses [4]-[6]. Globally it's often observed that carbon is abundant element in astrophysical sits having the atmosphere. Emission lines of C-like

*Corresponding Author: Nahed Hosny Wahba, Department of Laser Applications in Metrology, Photochemistry, and Agriculture, National Institute of Laser Enhanced Sciences, Cairo University, Giza, 12613, Egypt

Email: nahedwahba77@gmail.com ions are functionalized at prosopopoeia of the solar, astrophysical and melting plasmas whose illustrating needed exact atomic calculations; where the soft X-ray and XUV regions most of the data was found[7], [8]; thus Electron Collisional Pumping was functionalized to generate soft X-ray lasers after pumping methods[9], [10].

The process of pumping was illustrated as following:

$$
X_{1}^{\mathrm{n}+}+\mathrm{e} \longrightarrow X_{\mathrm{u}}^{\mathrm{n}+}
$$

where $X_{1}{ }^{\mathrm{n}+}$ is a n-frequencies of atom ionization of the element $\mathrm{X}$ that pumping occurrence from lower level "l" to an excited level " $u$ " in the same element atoms.

Theoretically there are more works done for computing the energy states, transition possibilities' and oscillator powers for $\mathrm{Zn}$ (XXV) [11]-[17]; while the gain for the same element not have 
more studies. The goal of this thesis is to utilize the atomic calculations such as energy states, oscillator powers and spontaneous radiative decay rates which calculated by using (FAC) program depending on Dirac equation for sixty nine thinstructure states to compute reduced populations and gain coefficients of C-like $\mathrm{Zn}$ excited states through a broad extent of electron densities $\left(10^{+20}\right.$ to $\left.10^{+23}\right)$ and at several electron temperatures $(700,800,900,1000,1100,1200,1300,1400 \&$ 1500).These calculations might support the experimentalists for generating soft $\mathrm{X}$-ray lasers.

\section{Calculations equations used for Gain Coefficient determination}

To calculate gain coefficient firstly energy levels, weighted oscillator strength and radiative rate for allowed transitions should be calculated; then the reduced population should calculated by solving coupled rate equation [18], [19]. After calculating the reduced population, it used to solve the Doppler broadening equation to obtain the gain coefficient.

Laser emission from $\mathrm{Zn}(\mathrm{XXV})$ ions plasma was investigated by studying the relation between several plasma temperatures and electron densities.

According to equation (1)

$$
\begin{aligned}
N_{u}\left[\sum_{l<u} A_{u l}+N_{e}\right. & \left.\left(\sum_{l<u} C_{u l}^{d}+\sum_{l>u} C_{u l}^{e}\right)\right] \\
= & N_{e}\left(\sum_{l<u} N_{l} C_{l u}^{e}+\sum_{l>u} N_{l} C_{l u}^{d}\right) \\
& +\sum_{l>u} N_{l} A_{l u}
\end{aligned}
$$

Since $N_{u}$ and $N_{l}$ are the fractional populations of levels $u$ and $l$ successively, $A_{u l}$ represents Einstein coefficient for spontaneous radiative decay from u to $1 ; N_{e}$ represents the electron density and $C_{l u}^{e}$ and $C_{u l}^{d}$ are the rate coefficients for collisional excitation and de-excitation successively. The actual population density $N_{u}$ of the $u^{\text {th }}$ state can be computed from relation (2) [20][21].

$$
C_{u l}^{d}=C_{l u}^{e}\left[\frac{g_{l}}{g_{u}}\right] \exp \left[\frac{\Delta E_{u l}}{k T_{e}}\right]
$$

Since $g_{l}$ and $g_{u}$ represents a statistical weights of lower and upper states, successively.

The electron impact excitation rates identified by the effective collision strengths $\gamma_{u l}$

[20]Where;

$$
C_{u l}^{d}=\frac{8.6287 * 10^{-6}}{\mathrm{gu}_{\mathrm{u}} \mathrm{T}_{\mathrm{e}}^{1 / 2}} \gamma_{l u}
$$

The measured population density $\mathrm{N}_{\mathrm{u}}$ of the $\mathrm{u}^{\text {th }}$ was calculated [20],

$$
N_{U}=N_{u} * N_{l}
$$

Since $N_{L}$ is the number of ions which achieved at the ionization stage $L[20]$,

$$
N_{L}=f_{L} N_{e} / Z_{\text {avg }}
$$

Since $N_{e}$ is the electron density, $Z_{\text {avg }}$ is the average degree of ionization and $f_{L}$ is the fractional abundance of the ionization levels were calculated [20]. Where the populations computed from Equation (1) is equal the unit;

$$
\sum_{U=1}^{69} \frac{N_{u}}{N_{l}}=1
$$

where the populations density calculated by Equation (1) is equal unit,

By computation the state's population density, the values $N_{u} / g_{u}$ and $N_{l} / g_{l}$ can be determined.

To prove that when inversion factor $(\mathrm{F}>0)$ gives positive gain equation (7) was used[22];

$$
F=\frac{g_{u}}{N_{u}}\left[\frac{N_{u}}{g_{u}}-\frac{N_{l}}{g_{l}}\right]
$$

Since $N_{u} / g_{u}$ and $N_{l} / g_{l}$ are the reduced populations of the upper state and lower state successively. Then Eq. (7) used to compute the gain coefficient $(\alpha)$ for Doppler broadening of the various transitions in the $\mathrm{Zn}(\mathrm{XXV})$ ion.

$$
\alpha_{u l}=\frac{\lambda_{l u}^{3}}{8 \pi}\left[\frac{M}{2 \pi K T_{l}}\right]^{1 / 2} A_{u l} N_{u} F
$$

Since $M$ is the ion mass, $\lambda_{l u}$ is the transition wavelength in $(\mathrm{nm})$, and $T_{l}$ is the ion temperature in $\mathrm{eV}$.

\section{Results and discussions}

\subsection{Energy states}

With utilizing (FAC) [23] energy state measures for the $1 \mathrm{~s}^{2}$ $2 \mathrm{~s}^{2} 2 \mathrm{pnl}(\mathrm{n}=3, \mathrm{l}=\mathrm{s}, \mathrm{p} \& \mathrm{~d})$ and $\mathrm{ml}(\mathrm{m}=4, \mathrm{l}=\mathrm{s}, \mathrm{p}, \mathrm{d} \& \mathrm{f})$ configurations in C-like $\mathrm{Zn}^{+30}$ was obtained, this data presented in Tables (1); which shows the 69 energy levels of transition configurations:

Table (2) presented the comparison between our calculations of energy levels for Zn (XXV) the theoretical calculations by Bhatia, Seely and Feldman [12] and the actual results computed by NIST [24].

In table (2), the calculated data for energy levels of Zn (XXV) comparing to experimental values in NIST at $\left(2 \mathrm{p}_{1 / 2} 2 \mathrm{p}_{3 / 2}\right)_{1}$ and $\left(2 \mathrm{p}_{1 / 2} 2 \mathrm{p}_{3 / 2}\right)_{2}$ by 0.05 and 0.04 successively; and it differs than the theoretical work of Bhatia at $\left(2 \mathrm{p}_{1 / 2} 2 \mathrm{p}_{3 / 2}\right)_{1}$ and $\left(2 \mathrm{p}_{1 / 2} 2 \mathrm{p}_{3 / 2}\right)_{2}$ by 0.05 Ryd and 0.04 Ryd successively also; which proved that our calculations are in well agreement with other works.

\subsection{Level population}

Where increasing the excited electrons in higher energy states than in ground state causes the production of Laser in the XUV and soft X-ray spectral area; 
Table 1: Energy states and definitions for Zn (XXV)

\begin{tabular}{|c|c|c|c|c|c|}
\hline index & $\begin{array}{c}\text { State } \\
\text { configuration }\end{array}$ & $\begin{array}{l}\text { Energy } \\
\text { in } \\
\text { (Ryd)* }\end{array}$ & Index & S tate configuration & $\begin{array}{l}\text { Energy } \\
\text { in } \\
\text { (Ryd)* }\end{array}$ \\
\hline 1 & $\left(2 p_{0}\right)_{0}$ & 0 & 36 & $\left(2 \mathrm{p}_{1 / 2} 4 \mathrm{p}_{\mathrm{s} / 2}\right)_{2}$ & 133.053 \\
\hline 2 & $\left(2 \mathrm{p}_{1 / 2} 2 \mathrm{pas}_{\mathrm{a}}\right)_{\mathrm{t}}$ & 1.3831 & 37 & $(2 \mathrm{p} / 24 \mathrm{pin}) 0$ & 133.075 \\
\hline 3 & $\left(2 \mathrm{pt} / 2 \mathrm{ps}_{2}\right)_{2}$ & 1.9445 & 38 & $\left(2 \mathrm{p} v 24 \mathrm{~s}_{1 / 2}\right)_{2}$ & 133.754 \\
\hline 4 & $\left(2 p_{2}\right)_{2}$ & 3.8985 & 39 & $\left(2 p_{12} 4 s_{1 / 2}\right)_{1}$ & 133.886 \\
\hline 5 & $\left(2 p_{0}\right)_{0}$ & 5.6409 & 40 & $\left(2 p_{1} / 24 d_{32}\right)_{2}$ & 133.979 \\
\hline 6 & $(2 \mathrm{pla} 3 \mathrm{~s}: 2) 0$ & 97.617 & 41 & $\left.2 \mathrm{p}: / 24 \mathrm{~d}_{5 / 2}\right)_{2}$ & 133.994 \\
\hline 7 & $(2 \mathrm{pla} 3 \mathrm{~s} / 2)_{\mathrm{t}}$ & 97.715 & 42 & $\left(2 \mathrm{p}_{1 / 2} 4 \mathrm{~d}_{\mathrm{s} / 2}\right)_{3}$ & 134.003 \\
\hline 8 & $\left(2 \mathrm{p}_{\mathrm{s} 2} 3 \mathrm{~s}: 2\right)_{2}$ & 99.576 & 43 & $\left(2 p_{1 / 2} 4 d_{32}\right)_{1}$ & 134.016 \\
\hline 9 & $(2 \mathrm{pgs} 3 \mathrm{~s} / 2) \mathrm{t}$ & 99.657 & 44 & $\left(2 p_{g / 2} 4 p_{12}\right)_{t}$ & 134.400 \\
\hline 10 & $(2 \mathrm{p}: 23 \mathrm{pia}) \mathrm{t}$ & 99.907 & 45 & $\left(2 p_{9 / 2} 4 p_{92}\right)_{3}$ & 134.424 \\
\hline 11 & $\left(2 \mathrm{p}_{1 / 2} 3 \mathrm{psa}_{2}\right)_{2}$ & 100.488 & 46 & $\left(2 p_{9 / 2} 4 p_{12}\right)_{2}$ & 134.440 \\
\hline 12 & $\left(2 \mathrm{p}_{1 / 2} 3 \mathrm{p}_{\mathrm{a}}\right):$ & 100.490 & 47 & $\left(2 \mathrm{p}_{\mathrm{S} / 2} 4 \mathrm{p}_{\mathrm{S}}\right)_{\mathrm{i}}$ & 134.447 \\
\hline 13 & $\left(2 \mathrm{p}_{1 / 2} 3 \mathrm{p}_{\mathrm{i}, 2}\right)_{0}$ & 100.568 & 48 & $\left(2 p_{1} / 2 f_{5 / 2}\right)_{3}$ & 134.873 \\
\hline 14 & $\left(2 p_{9 / 2} 3 p_{a 2}\right)_{t}$ & 102.038 & 49 & $\left(2 \mathrm{p} / 24 f_{s / 2}\right)_{2}$ & 134.921 \\
\hline 15 & $\left(2 \mathrm{p}_{9 / 2} 3 \mathrm{p}_{92}\right)_{3}$ & 102.206 & 50 & $\left(2 \mathrm{p}_{1 / 2} 4 f_{7 / 2}\right)_{3}$ & 134.996 \\
\hline 16 & $\left(2 \mathrm{p}_{\mathrm{s} / 2} 3 \mathrm{p}_{\mathrm{i} / 2}\right)_{\mathrm{t}}$ & 102.230 & 51 & $\left(2 \mathrm{p} / 24 f_{7 / 2}\right)_{4}$ & 135.019 \\
\hline 17 & $\left(2 \mathrm{p}_{\mathrm{g} / 2} 3 \mathrm{p}_{\mathrm{i}, 2}\right)_{2}$ & 102.275 & 52 & $\left(2 p_{9 / 2} 4 p_{92}\right)_{2}$ & 135.251 \\
\hline 18 & $(2 \mathrm{p}: 23 \mathrm{dsa})_{2}$ & 102.289 & 53 & $(2 \mathrm{ps} / 24 \mathrm{psa})_{0}$ & 135.496 \\
\hline 19 & $\left(2 \mathrm{p}_{9 / 2} 3 \mathrm{p}_{9 \mathrm{a}}\right)_{2}$ & 102.812 & 54 & $\left(2 p_{s / 2} 4 d_{s 2}\right)_{4}$ & 135.854 \\
\hline 20 & $\left(2 \mathrm{p}_{2} / 3 \mathrm{~d}_{52}\right)_{3}$ & 102.842 & 55 & $\left(2 p_{a / 2} 4 d_{3 a}\right)_{2}$ & 135.866 \\
\hline 21 & $\left(2 \mathrm{pt} / 23 \mathrm{~d}_{50}\right)_{2}$ & 102.979 & 56 & 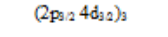 & 135.928 \\
\hline 22 & $\left(2 \mathrm{p}_{12} 3 \mathrm{~d}_{32}\right)$ & 103.056 & 57 & $\left(2 \mathrm{p}_{\mathrm{s} / 2} 4 \mathrm{~d}_{\mathrm{s} / 2}\right)_{2}$ & 135.993 \\
\hline 23 & $\left(2 p_{9 / 2} 3 p_{a, 2}\right)_{0}$ & 103.722 & 58 & $\left(2 p_{9 / 2} 4 d_{32}\right)_{t}$ & 136.004 \\
\hline 24 & $\left(2 \mathrm{p}_{9 / 2} 3 \mathrm{~d}_{s a}\right)_{4}$ & 104.441 & 59 & $\left(2 \mathrm{p}_{9 / 2} 4 d_{92}\right)_{0}$ & $136 \cap 05$ \\
\hline 25 & $\left(2 \mathrm{p}_{32} 3 \mathrm{~d}_{\mathrm{s} / 2}\right)_{2}$ & 104.474 & 60 & $\left(2 \mathrm{p}_{\mathrm{g} / 2} 4 \mathrm{~d}_{\mathrm{s} 2}\right)_{3}$ & 136.192 \\
\hline 26 & $\left(2 \mathrm{p}_{3 / 2} 3 \mathrm{~d}_{5 / 2}\right)_{3}$ & 104.717 & 61 & $\left(2 \mathrm{p}_{9 / 2} 4 \mathrm{~d}_{52}\right)_{1}$ & 136.232 \\
\hline 27 & $\left(2 \mathrm{p}_{\mathrm{n} 2} 3 \mathrm{~d}_{\mathrm{s} / 2}\right)_{2}$ & 104.892 & 62 & $\left(2 p_{a / 2} 4 f_{s / 2}\right)_{t}$ & 136.421 \\
\hline 28 & $\left(2 \mathrm{p}_{3 / 2} 3 \mathrm{~d}_{\mathrm{s} / 2}\right)_{\mathrm{t}}$ & 104.907 & 63 & $\left(2 p_{9 / 2} 4 f_{1 / 2}\right)_{4}$ & 136.450 \\
\hline 29 & $\left(2 \mathrm{p}_{32} 3 \mathrm{~d}_{3 / 2}\right)_{0}$ & 104.921 & 64 & $\left(2 \mathrm{ps}_{\mathrm{s}} 4 \mathrm{f}_{\mathrm{s} / 2}\right)_{2}$ & 136.475 \\
\hline 30 & $\left(2 \mathrm{p}_{12} 3 \mathrm{~d}_{5 / 2}\right)_{3}$ & 105.508 & 65 & $\left(2 f_{j / 2} 4 f_{/ 2}\right)_{3}$ & 136.488 \\
\hline 31 & $\left(2 \mathrm{p}_{32} 3 \mathrm{~d}_{\mathrm{s} / 2}\right)_{t}$ & 105.564 & 66 & $\left(2 \mathrm{p}_{\mathrm{a} / 2} 4 \mathrm{f}_{1 / 2}\right)_{\mathrm{s}}$ & 136.508 \\
\hline 32 & $(2 \mathrm{p}, 24 \mathrm{~s}, 2) \mathrm{b}$ & 131.880 & 67 & $\left(2 \mathrm{pg}_{/ 2} 4 \mathrm{fs}_{\mathrm{g} / 2}\right)_{4}$ & 136.522 \\
\hline 33 & $\left(2 p_{1 / 2} 4 s: 2\right)$ & 131.914 & 68 & $\left(2 \mathrm{p}_{\mathrm{i}, 2} 4 \mathrm{f}_{\mathrm{s} / 2}\right)_{\mathrm{i}}$ & 136.540 \\
\hline 34 & $\left(2 \mathrm{p}_{12} 4 \mathrm{p}_{1 / 2}\right)_{t}$ & 132.702 & 69 & $\left(2 p_{9 / 2} 4 f_{1 / 2}\right)_{2}$ & 136.580 \\
\hline 35 & $\left(2 p_{1 / 2} 4 p_{9 / 2}\right)_{t}$ & 133.037 & & & \\
\hline
\end{tabular}

* Ryd is Rydberg constant

\section{normal distribution population inversion}
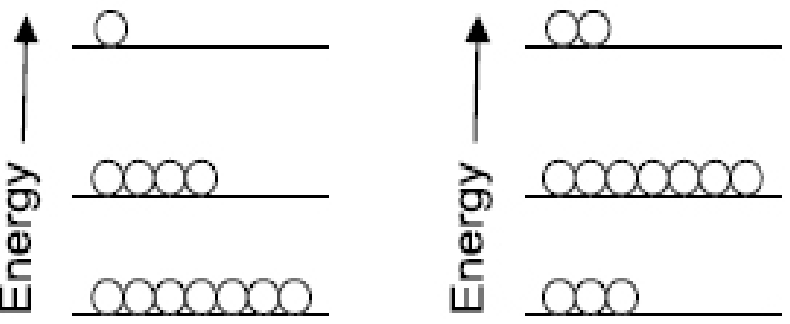

Schematic diagram of population inversion (Source of figure: https://spie.org/publications/fg08_p94_lasers?SSO=1)

\begin{tabular}{|c|c|c|c|c|}
\hline index & $\begin{array}{c}\text { State } \\
\text { Configuration }\end{array}$ & $\begin{array}{l}\text { Our calculation } \\
\text { (FAC)(a) }\end{array}$ & $S S^{(\theta)}$ & $\mathrm{NIS}^{(\mathrm{s})}$ \\
\hline 1 & $(2 \mathrm{p} 0)_{0}$ & 0 & 0 & 0 \\
\hline 2 & $\left(2 \mathrm{p}_{12} 2 \mathrm{p}_{3 / 2}\right):$ & 1.3831 & 1.4372 & 1.4370 \\
\hline 3 & $\left(2 \mathrm{p}_{12} 2 \mathrm{p}_{\mathrm{p} / 2}\right)_{2}$ & 1.9445 & 1.9866 & 1.9870 \\
\hline 4 & $\left(2 \mathrm{p}_{2}\right)_{2}$ & 3.8985 & 3.9122 & ... \\
\hline 5 & $\left(2 \mathrm{p}_{0}\right)_{0}$ & 5.6409 & 53061 & \\
\hline 6 & $\left(2 \mathrm{p}: 23 \mathrm{~s}_{12}\right) \mathrm{b}$ & 97.617 & 98.206 & $\ldots$ \\
\hline 7 & $\left(2 p_{12} 3 s_{12}\right)$ & 97.715 & 98.306 & $\ldots$ \\
\hline 8 & $\left(2 p_{s / 2} 3 s_{12}\right)$ & 99.576 & 100.152 & \\
\hline 9 & $\left(2 p_{92} 3 s_{12}\right)$ & 99.657 & 100.395 & $\cdots$ \\
\hline 10 & $\left(2 p_{12} 3 p_{1} 2\right)_{t}$ & 99.907 & 100.154 & $\ldots$ \\
\hline 11 & $\left(2 \mathrm{p}_{12} 3 \mathrm{p}_{\mathrm{s} / 2}\right)_{2}$ & 100.488 & 101.035 & $\ldots$ \\
\hline 12 & $\left(2 \mathrm{p}_{\mathrm{p} / 2} 3 \mathrm{p}_{\mathrm{s} / 2}\right)_{\mathrm{t}}$ & 100.490 & 101.040 & $\ldots$ \\
\hline 13 & $\left(2 p_{12} 3 p_{12}\right)_{0}$ & 100.568 & 101.143 & $\ldots$ \\
\hline 14 & $\left(2 \mathrm{p}_{32} 3 \mathrm{p}_{9 / 2}\right)$ & 102.038 & 102.522 & \\
\hline 15 & $\left(2 \mathrm{p}_{22} 3 \mathrm{p}_{12 / 2}\right)_{3}$ & 102.206 & 102.670 & $\ldots$ \\
\hline 16 & $\left(2 \mathrm{p}_{32} 3 \mathrm{p}_{1 / 2}\right)_{\mathrm{t}}$ & 102.230 & 102.752 & $\ldots$ \\
\hline 17 & $\left(2 \mathrm{p}_{32} 3 \mathrm{p}_{12}\right)_{2}$ & 102.275 & 102.728 & $\ldots$ \\
\hline 18 & $\left(2 p_{1 / 2} 3 d_{1 / 2}\right)_{2}$ & 102.289 & 103.503 & $\ldots$ \\
\hline 19 & $\left(2 \mathrm{p}_{12} 3 \mathrm{p}_{\mathrm{p} / 2}\right)_{2}$ & 102.812 & 102.847 & \\
\hline 20 & $\left(2 \mathrm{p}_{1 / 2} 3 \mathrm{~d}_{5 / 2}\right)_{3}$ & 102.842 & 104.155 & $\ldots$ \\
\hline 21 & $\left(2 \mathrm{p}_{12} 3 \mathrm{~d}_{5 / 2}\right)_{2}$ & 102.979 & 103.399 & $\cdots$ \\
\hline 22 & $\left(2 \mathrm{p}_{2} 3 \mathrm{~d}_{\mathrm{y}_{2}}\right)_{\mathrm{t}}$ & 103.056 & 103.443 & \\
\hline 23 & $\left(2 \mathrm{p}_{32} 3 \mathrm{p}_{32}\right)_{0}$ & 103.722 & 103.589 & \\
\hline 24 & $\left(2 \mathrm{p}_{32} 3 \mathrm{~d}_{52}\right)_{4}$ & 104.441 & 104.934 & ... \\
\hline 25 & $\left(2 \mathrm{p}_{32} 3 \mathrm{~d}_{32}\right)_{2}$ & 104.474 & 104.973 & $\ldots$ \\
\hline 26 & $\left(2 p_{32} 3 d_{52} b_{3}\right.$ & 104.717 & 105.227 & $\ldots$ \\
\hline 27 & $\left(2 p_{s 2} 3 d_{s 2}\right)_{2}$ & 104.892 & 105.415 & $\ldots$ \\
\hline 28 & $\left(2 \mathrm{p}_{92} 3 \mathrm{~d}_{32}\right)$ & 104.907 & 105.382 & $\cdots$ \\
\hline 29 & $\left(2 \mathrm{p}_{32} 3 \mathrm{~d}_{32}\right)_{\mathrm{b}}$ & 104.921 & 105.390 & $\ldots$ \\
\hline
\end{tabular}

Thus the process of the reduced population densities was computed for sixty nine thin structure states starting from $1 \mathrm{~s}^{2} 2 \mathrm{~s}^{2}$ $2 \mathrm{pnl}(\mathrm{n}=3, \mathrm{l}=\mathrm{s}, \mathrm{p} \& \mathrm{~d})$ and $\mathrm{ml}(\mathrm{m}=4, \mathrm{l}=\mathrm{s}, \mathrm{p}, \mathrm{d} \& \mathrm{f})$ configurations. The determination was done by applying the coupled rate Eq. (1) simultaneously using MATLAB version 7.10.0 (R2010a) computer program [25][17].

Figure (1 to 4$)$ illustrate the reduced population for states $\left(2 \mathrm{p}_{3 / 2} 3 \mathrm{~s}_{1 / 2}\right)_{2},\left(2 \mathrm{p}_{1 / 2} 3 \mathrm{p}_{3 / 2}\right)_{1,}\left(2 \mathrm{p}_{3 / 2} 3 \mathrm{p}_{3 / 2}\right)_{3},\left(2 \mathrm{p}_{3 / 2} 3 \mathrm{p}_{3 / 2}\right)_{1,}\left(2 \mathrm{p}_{3 / 2} 3 \mathrm{~d}_{5 / 2}\right)_{4}$, and $\left(2 \mathrm{p}_{3 / 2} 3 \mathrm{~d}_{3 / 2}\right)_{3}$ at various temperatures $(800,900,1000,1100) \mathrm{eV}$; so it can explain the behavior of states populations' density for several ions; where at low electron densities the reduced population densities are proportional to the electron densities, and the excitation process for an excited state is followed immediately by radiation decay. These results were agreed with the results of Feldman et.al. [11,17,24]. At electron density $10^{+19}$ various peaks were appeared; which means that radiative transitions dominant the de-excitation due its higher energy and fast decay time. 


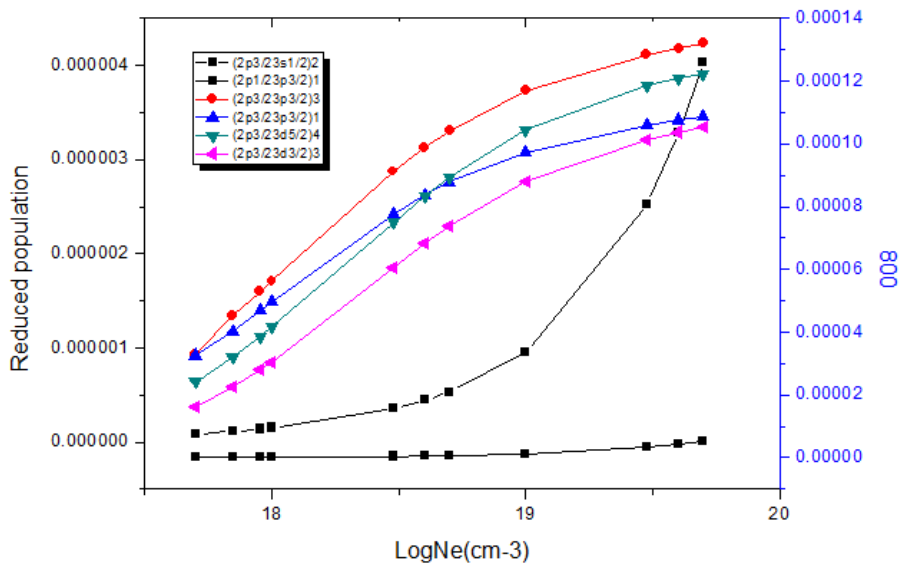

Figure 1: Reduced population of $\mathrm{Zn}^{+30}$ states at electron temperature $800 \mathrm{eV}$.

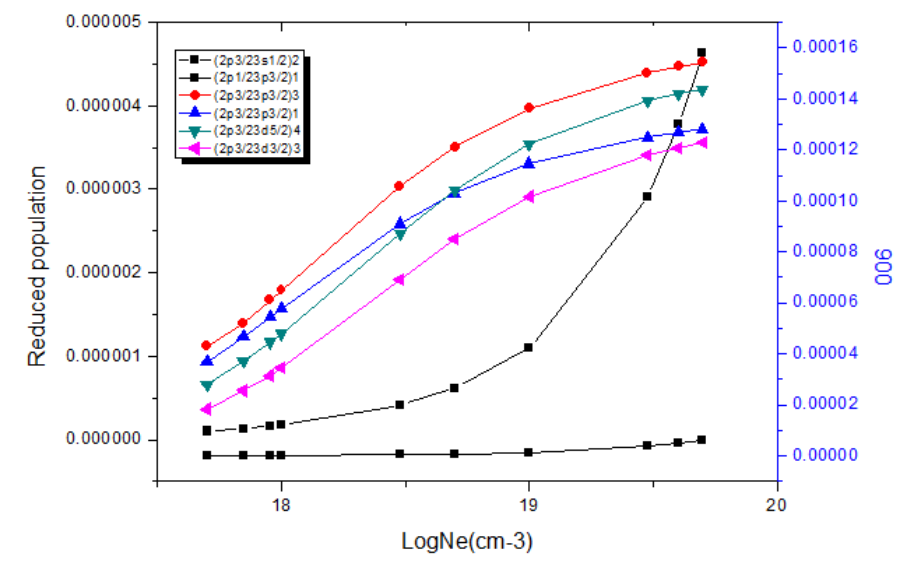

Figure 2: Reduced population of $\mathrm{Zn}^{+30}$ states at electron temperature $900 \mathrm{eV}$.

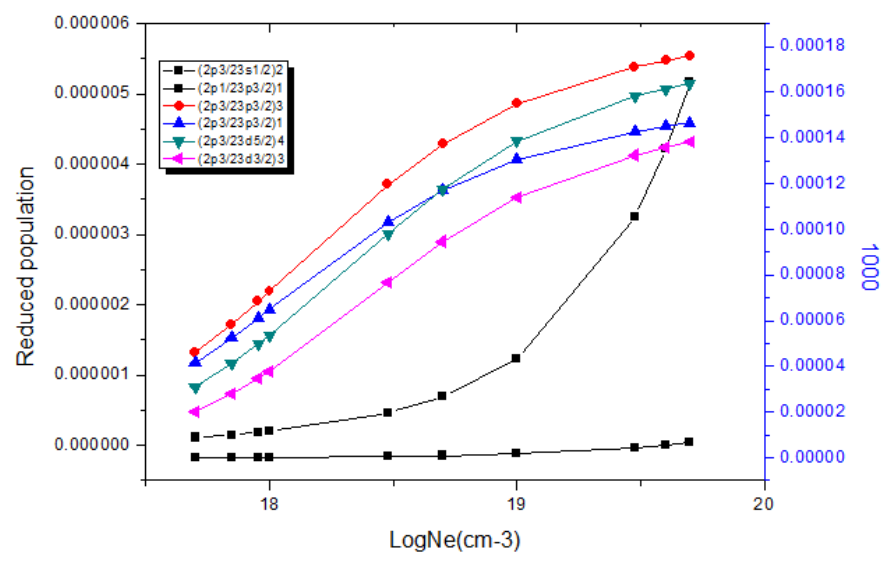

Figure 3: Reduced population of $\mathrm{Zn}^{+30}$ states at electron temperature $1000 \mathrm{eV}$.
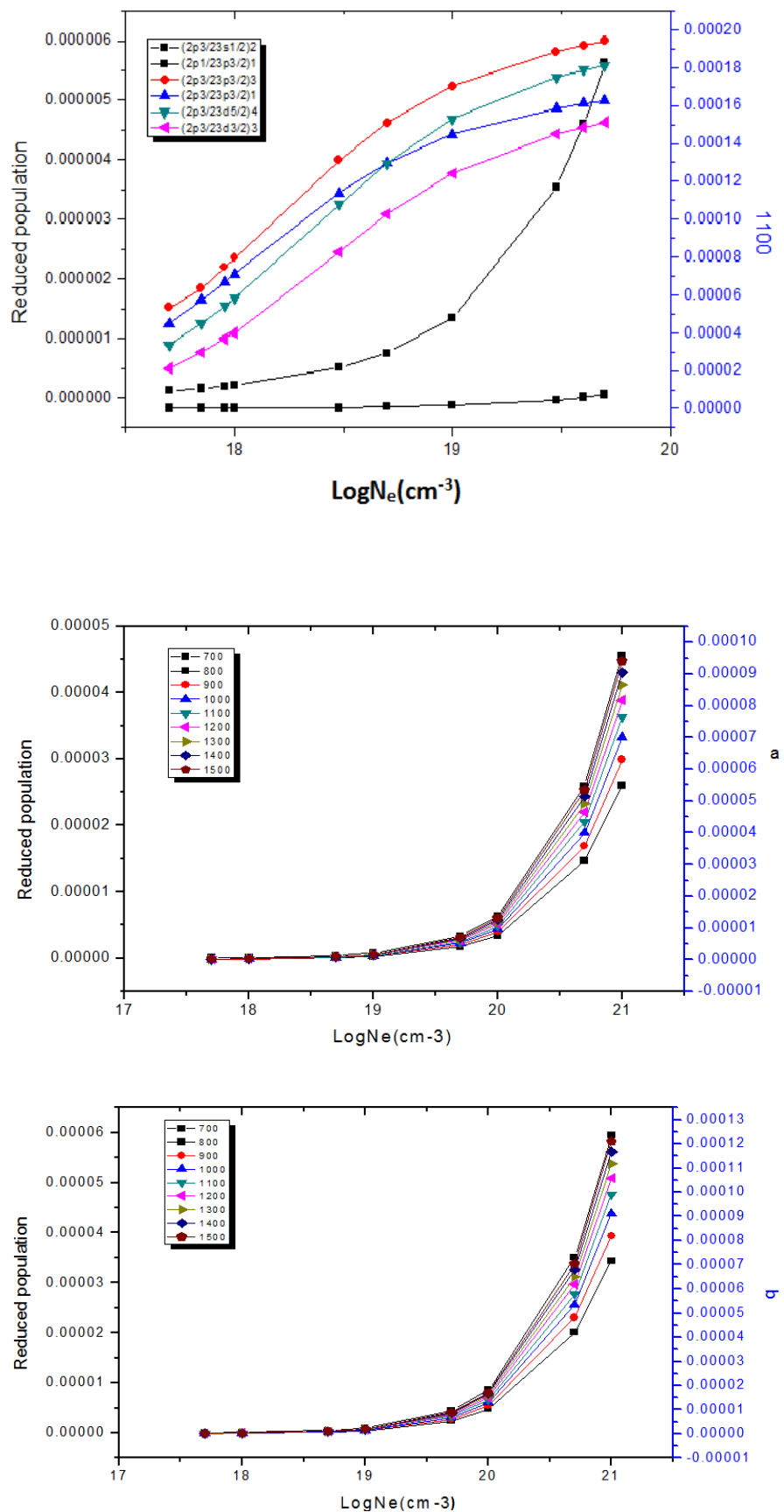

Figure 5: Reduced population of level (a) $\left(2 \mathrm{p}_{12} 3 \mathrm{p}_{12}\right)_{1}$, (b) $\left(2 \mathrm{p}_{122} 3 \mathrm{p}_{32}\right)_{1}$ for $\mathrm{Zn}(\mathrm{XXV})$ after electron collisional pumping as a function of the electron density at temperatures $(700,800,900,1000,1100,1200,1300,1400 \& 1500) \mathrm{eV}$.

\subsection{Radiative lifetime}


atomic transfer probability is related to the life time $u$ of an exited state
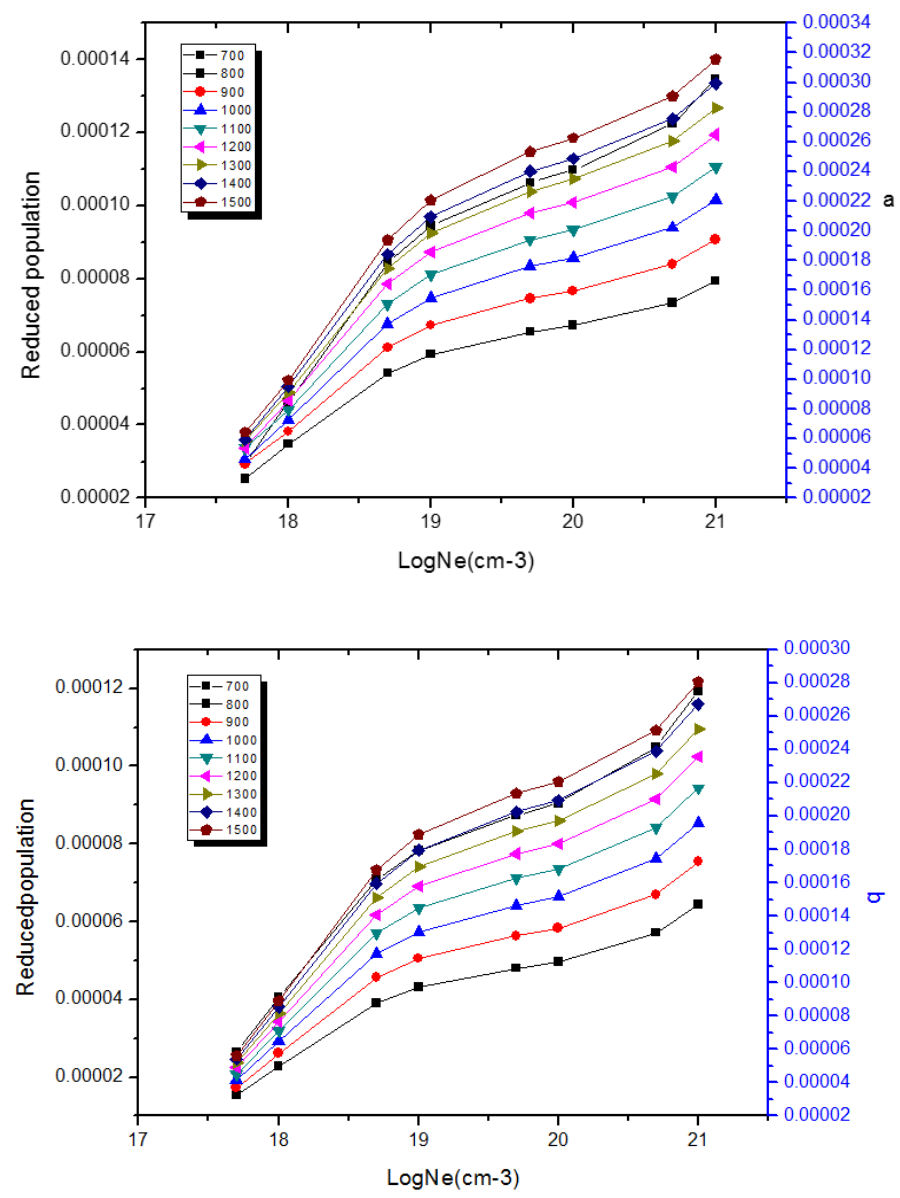

Figure 6: Reduced population of level (a) $\left(2 \mathrm{p}_{312} 3 \mathrm{p}_{312}\right)_{3}$, (b) $\left(2 \mathrm{p}_{312} 3 \mathrm{p}_{312}\right)_{1}$ for $\mathrm{Zn}(\mathrm{XXV})$ as a function of the electron density at different electron temperatures $(700,800$, $900,1000,1100,1200,1300,1400 \& 1500) \mathrm{eV}$.

$$
\tau_{u}=\frac{1}{\sum_{l} A_{u l}}
$$

Table 3. Illustrate the results of $\left(2 \mathrm{p}_{1 / 2} 3 \mathrm{~d}_{3 / 2}\right)_{2}--\left(2 \mathrm{p}_{1 / 2} 3 \mathrm{p}_{3 / 2}\right)_{1}$, $\left(2 \mathrm{p}_{3 / 2} 3 \mathrm{~d}_{5 / 2}\right)_{4}-\left(2 \mathrm{p}_{1 / 2} 3 \mathrm{p}_{3 / 2}\right)_{1}$ and $\left(2 \mathrm{p}_{3 / 2} 3 \mathrm{~d}_{5 / 2}\right)_{4}-\left(\left(2 \mathrm{p}_{1 / 2} 3 \mathrm{p}_{3 / 2}\right)_{2}\right.$ radiative life time is longer than the lifetime of the lower state.

\begin{tabular}{lll}
\hline Configuration & $\tau_{u}(\mathrm{sec})$ & $\tau_{l}(\mathrm{sec})$ \\
& & \\
\hline$\left(2 \mathrm{p}_{3 / 2} 3 \mathrm{p}_{3 / 2}\right)_{3}--\left(2 \mathrm{p}_{1 / 2} 3 \mathrm{p}_{1 / 2}\right)_{1}$ & $8.926 \mathrm{e}-10$ & $7.495 \mathrm{e}-10$ \\
$\left(2 \mathrm{p}_{1 / 2} 3 \mathrm{~d}_{3 / 2}\right)_{2}--\left(2 \mathrm{p}_{1 / 2} 3 \mathrm{p}_{3 / 2}\right)_{1}$ & $9.894 \mathrm{e}-10$ & $3.493 \mathrm{e}-12$ \\
$\left(2 \mathrm{p}_{3 / 2} 3 \mathrm{p}_{3 / 2}\right)_{1}-\left(2 \mathrm{p}_{1 / 2} 3 \mathrm{p}_{3 / 2}\right)_{1}$ & $2.578 \mathrm{e}-13$ & $3.493 \mathrm{e}-12$ \\
$\left(2 \mathrm{p}_{3 / 2} 3 \mathrm{~d}_{5 / 2}\right)_{4}-\left(2 \mathrm{p}_{1 / 2} 3 \mathrm{p}_{3 / 2}\right)_{1}$ & $2.104 \mathrm{e}-9$ & $3.493 \mathrm{e}-12$ \\
$\left(2 \mathrm{p}_{3 / 2} 3 \mathrm{~d}_{5 / 2}\right)_{4}-\left(\left(2 \mathrm{p}_{1 / 2} 3 \mathrm{p}_{3 / 2}\right)_{2}\right.$ & $2.104 \mathrm{e}-9$ & $2.282 \mathrm{e}-10$ \\
$\left(2 \mathrm{p}_{3 / 2} 3 \mathrm{~d}_{3 / 2}\right)_{3}-\left(2 \mathrm{p}_{1 / 2} 3 \mathrm{~d}_{3 / 2}\right)_{2}$ & $5.597 \mathrm{e}-14$ & $9.894 \mathrm{e}-10$ \\
\hline
\end{tabular}

\subsection{Inversion factor}

According to equation (7) the reduced population for lower states and upper states was calculated and demonstrate in the equation to calculate the inversion factor and it's found that the inversion factor is larger than zero. By using electron collisional pumping process the pumping quanta can be transferred to other state as a result of collision process, and this cause population inversion from the upper states to the lower states; whence this population inversion achieved appositive gain via $\mathrm{F}>0[21]$.

\subsection{Gain coefficient}

The gain process is the measure of the part of medium energy transferred to the emitted radiation which causes the amplification of the emitted radiation leading to strength optical power.

To calculate the gain the MATlab version the program was used to solve the coupled rate equation; this by using $A_{u l}$ (spontaneous decay rates), $\mathrm{C}_{\mathrm{lu}}$ (electron collisional excitation rate coefficients) and $\mathrm{C}_{\mathrm{ul}}^{\mathrm{d}}$ (electron collisional deexcitation rate coefficients).

$$
>0 \text {. }
$$

Finally the Doppler broadening equation was solved for various transitions to give the gain coefficient; then by plotting the relation between gain and electron density at different temperature to obtain the most intense laser transitions.

The figures $(7,8,9,10 \& 11)$ illustrates the proportional relation between gain and electron density; and also have proportional relation between gain and temperature. According to the collected data it's found that the largest gain occur at temperature $(1100 \mathrm{eV})$ which give gain height of $\left(13.522 \mathrm{~cm}^{-1}\right)$ at wavelength $(50 \mathrm{~nm})$; this transition is at $\left(2 \mathrm{p}_{3 / 2} 3 \mathrm{p}_{3 / 2}\right)_{1}-\left(2 \mathrm{p}_{1 / 2} 3 \mathrm{p}_{3 / 2}\right)_{1}$ which refers to them by $(16<>9)$. The smallest gain occur at temperature $(800 \mathrm{eV})$ which give gain height of $\left(2.5530 \mathrm{~cm}^{-1}\right)$ at wavelength $(22.79 \mathrm{~nm})$; this gain transition at $\left(2 \mathrm{p}_{3 / 2} 3 \mathrm{~d}_{5 / 2}\right)_{4}$ $\left(2 \mathrm{p}_{1 / 2} 3 \mathrm{p}_{3 / 2}\right)_{1}$ which describe them as $(22<>10)$; the gain of these transition at $(22<>10)$ and at $(16<>9)$ was plotted against electron densities at different temperatures. See Figure (11).

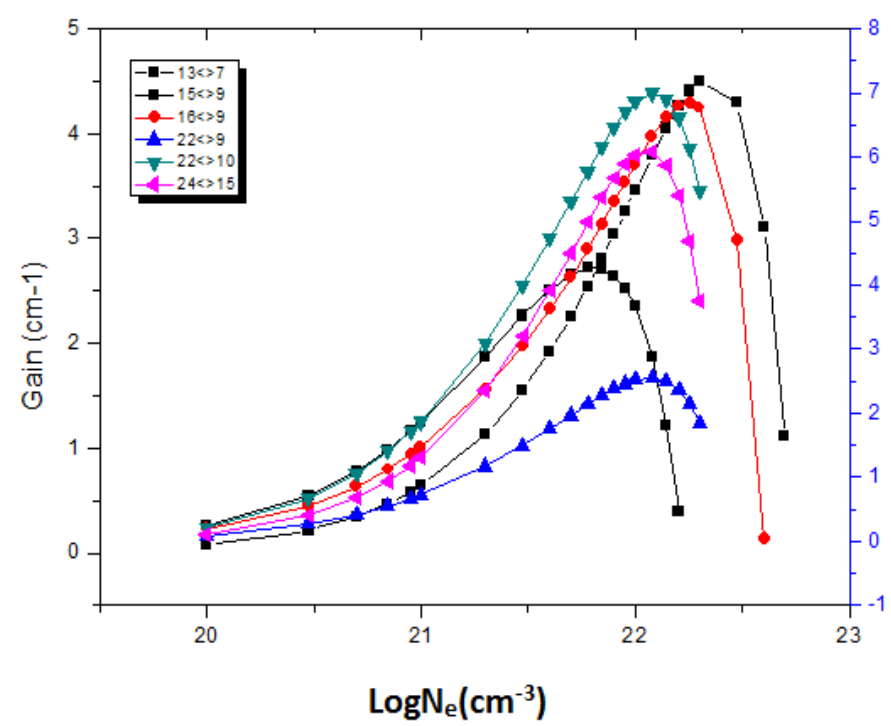

Figure 7: Electron density versus Gain coefficient at temperature $800 \mathrm{eV}$. 


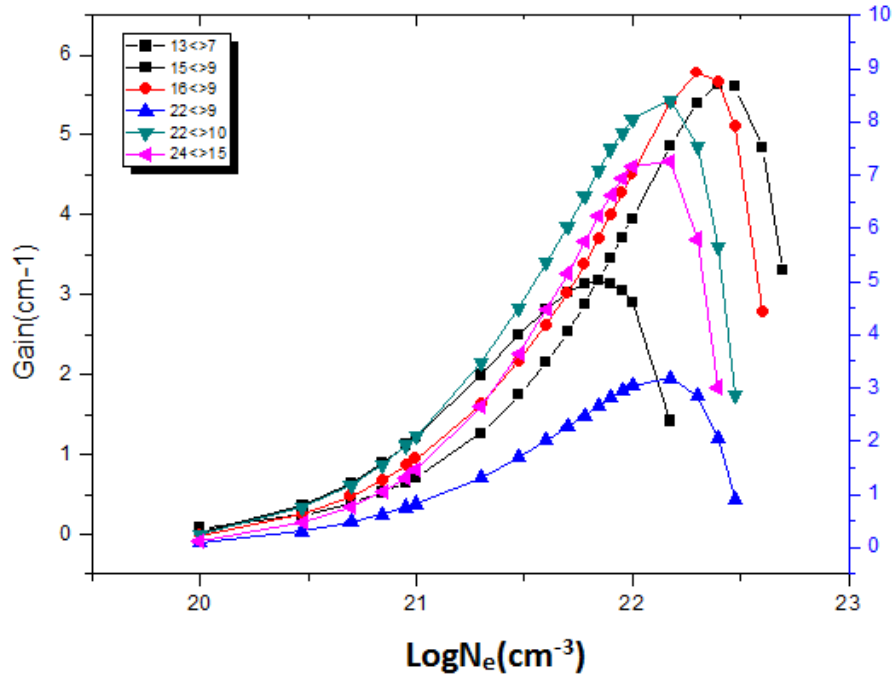

Figure 8: Electron density versus Gain coefficient at temperature $900 \mathrm{eV}$.

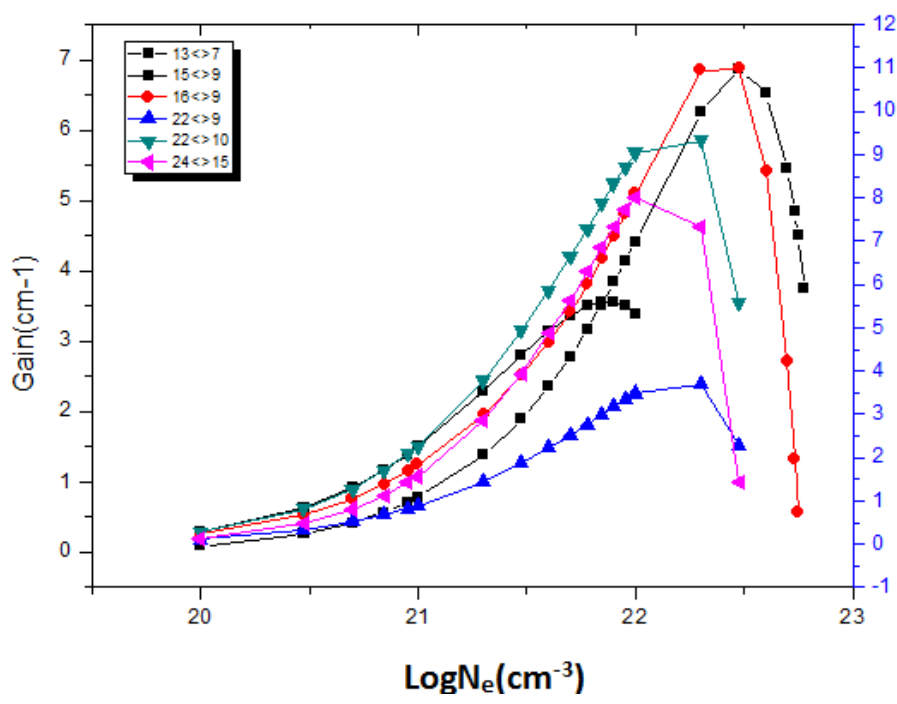

Figure 9: Electron density versus Gain coefficient at temperature $1000 \mathrm{eV}$.

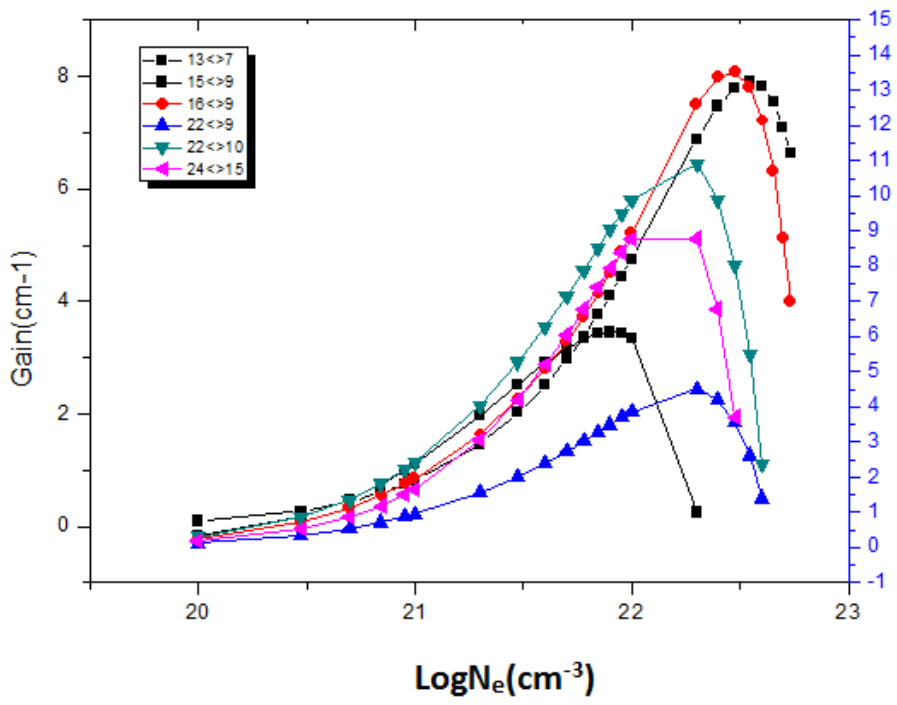

Figure 10: Electron density versus Gain coefficient at temperature $1100 \mathrm{eV}$.
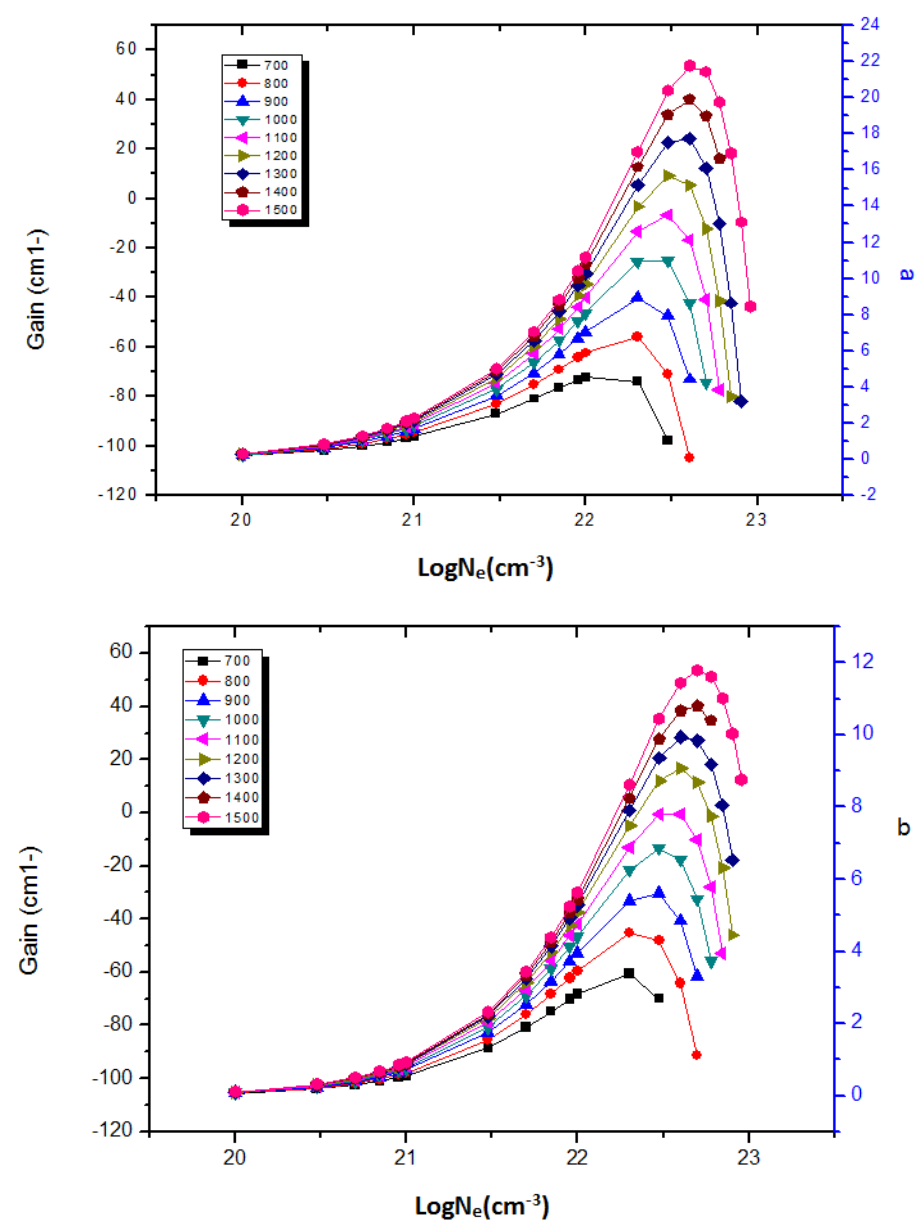

Table 4: configuration states, wavelength and maximum gain coefficient at various temperatures.

\begin{tabular}{|c|c|c|c|c|c|c|c|c|c|c|}
\hline \multirow{2}{*}{ Configuration } & \multirow{2}{*}{$\lambda(\mathbf{n m})$} & \multicolumn{70}{|c|}{ Gain(o)(cm $\mathbf{l})$} \\
\cline { 3 - 11 } & & 700 & 800 & 900 & 1000 & 1100 & 1200 & 1300 & 1400 & 1500 \\
\hline$\left(2 \mathrm{p}_{3 / 2} 3 \mathrm{p}_{3 / 2}\right)_{3}--\left(2 \mathrm{p}_{1 / 2} 3 \mathrm{p}_{1 / 2}\right)_{1}$ & 35.34 & 3.372 & 4.504 & 5.635 & 6.866 & 7.909 & 9.067 & 9.979 & 10.814 & 11.795 \\
\hline$\left(2 \mathrm{p}_{1 / 2} 3 \mathrm{~d}_{3 / 2}\right)_{2}--\left(2 \mathrm{p}_{1 / 2} 3 \mathrm{p}_{3 / 2}\right)_{1}$ & 50.4 & 3.409 & 4.266 & 5.006 & 5.595 & 6.118 & 6.560 & 6.899 & 7.156 & 7.387 \\
\hline$\left(2 \mathrm{p}_{3 / 2} 3 \mathrm{p}_{3 / 2}\right)_{1}-\left(2 \mathrm{p}_{1 / 2} 3 \mathrm{p}_{3 / 2}\right)_{1}$ & 50 & 4.811 & 6.847 & 8.918 & 10.977 & 13.522 & 15.676 & 17.869 & 19.888 & 21.82 \\
\hline$\left(2 \mathrm{p}_{3 / 2} 3 \mathrm{~d}_{5 / 2}\right)_{4}-\left(2 \mathrm{p}_{1 / 2} 3 \mathrm{p}_{3 / 2}\right)_{1}$ & 22.79 & 1.938 & 2.553 & 3.182 & 3.711 & 4.504 & 5.144 & 5.811 & 6.438 & 7.173 \\
\hline$\left(2 \mathrm{p}_{3 / 2} 3 \mathrm{~d}_{5 / 2}\right)_{4}-\left(2 \mathrm{p}_{1 / 2} 3 \mathrm{p}_{3 / 2}\right)_{2}$ & 22.8 & 5.469 & 6.998 & 8.410 & 9.327 & 10.919 & 12.19 & 13.325 & 14.229 & 15.226 \\
\hline$\left(2 \mathrm{p}_{3 / 2} 3 \mathrm{~d}_{3 / 2}\right)_{3}-\left(2 \mathrm{p}_{1 / 2} 3 \mathrm{~d}_{3 / 2}\right)_{2}$ & 36.9 & 4.685 & 6.077 & 7.258 & 8.017 & 6.788 & 10.381 & 10.978 & 11.526 & 12.068 \\
\hline & & & & & & & & & \\
\hline
\end{tabular}

\section{Conclusions}




\section{Conflict of Interest}

The authors declare no conflict of interest.

\section{Acknowledgment}

The authors thanks both Prof. Dr. Souad ElFeky, Dr. Nagy Emara, and NILES their promotion and support.

\section{References:}

[1] William.T.Silfvast, Cambridge University press, second edi, 2000.

[2] B.N. Wellegehausen, B., Eichmann, H., Meyer, S., Momma, C., Mossavi, K., Welling, H., \&Chichkov, "Generation of coherent VUV and XUV radiation. In ICONO'95: Fundamentals of Laser-Matter Interaction.," International Society for Optics and Photonics., 2796, 132-139, 1996.

[3] A. Verma, R. Kumar, A. Parashar, "Enhanced thermal transport across a bicrystalline graphene-polymer interface: an atomistic approach," Physical Chemistry Chemical Physics, 21(11), 6229-6237, 2019. doi: 10.1039/C9CP00362B

[4] R.E. King, G.J. Pert, S.P. McCabe, P.A. Simms, A.G. MacPhee, C.L.S. Lewis, R. Keenan, R.M.N. O’Rourke, G.J. Tallents, S.J. Pestehe, "Saturated x-ray lasers at 196 and $73 \AA$ pumped by a picosecond traveling-wave excitation," Physical Review A, 64(5), 53810, 2001. doi: 10.1103/PhysRevA.64.053810

[5] A. V Vinogradov, I.I. Sobel'man, E.A. Yukov, "Population inversion of transitions in neon-like ions," Soviet Journal of Quantum Electronics, 7(1), 32, 1977.

[6] B.A. Norton, N.J. Peacock, "Population inversion in laser-produced plasmas by pumping with opacity-broadened lines," Journal of Physics B: Atomic and Molecular Physics, 8(6), 989, 1975.

[7] G. Tachiev, C.F. Fischer, "Breit-Pauli energy levels and transition rates for the carbonlike sequence," Canadian Journal of Physics, 79(7), 955-976, 2001. doi: 10.1139/p01-059

[8] K.M. Aggarwal, F.P. Keenan, A.Z. Msezane, "Oscillator strengths for transitions in C-like ions between K XIV and Mn XX," Astronomy \& Astrophysics, 401(1), 377-383, 2003.

[9] V.A. Bhagavatula, "Soft x-ray population inversion by resonant photoexcitation in multicomponent laser plasmas," Journal of Applied Physics, 47(10), 4535-4537, 1976.

[10] J. Nilsen, P. Beiersdorfer, S.R. Elliott, T.W. Phillips, B.A. Bryunetkin, V.M. Dyakin, T.A. Pikuz, A.Y. Faenov, S.A. Pikuz, S. Von Goeler, "Measurement of the Ly- $\alpha \mathrm{Mg}$ resonance with the $2 \mathrm{~s} \rightarrow 3 \mathrm{p}$ Ne-like Ge line," Physical Review A, 50(3), 2143, 1994.

[11] U. Feldman, G.A. Doschek, J.F. Seely, A.K. Bhatia, "Short wavelength laser calculations for electron pumping in $\mathrm{Be} \mathrm{I}$ and $\mathrm{BI}$ isoelectronic sequences (18 $\leq$ Z $\leq 36)$," Journal of Applied Physics, 58(8), 2909-2915, 1985.

[12] A.K. Bhatia, J.F. Seely, U. Feldman, "Atomic data and spectral line intensities for the carbon isoelectronic sequence (Ar XIII through Kr XXXI)," Atomic Data and Nuclear Data Tables, 36(3), 453-494, 1987, doi:https://doi.org/10.1016/0092-640X(87)90012-X.

[13] A. Verma, A. Parashar, "Molecular dynamics based simulations to study the fracture strength of monolayer graphene oxide," Nanotechnology, 29(11), 115706, 2018, doi:10.1088/1361-6528/aaa8bb.

[14] A. Verma, A. Parashar, "Molecular dynamics based simulations to study failure morphology of hydroxyl and epoxide functionalised graphene," Computational Materials Science, 143, 15-26, 2018.

[15] V. Singla, A. Verma, A. Parashar, "A molecular dynamics based study to estimate the point defects formation energies in graphene containing STW defects," Materials Research Express, 6(1), 15606, 2018. doi: 10.1088/20531591

[16] A. Verma, A. Parashar, M. Packirisamy, "Atomistic modeling of graphene/hexagonal boron nitride polymer nanocomposites: a review," Wiley Interdisciplinary Reviews: Computational Molecular Science, 8(3), e1346, 2018. doi: 10.1088/25192018

[17] U. Feldman, J.F. Seely, G.A. Doschek, A.K. Bhatia, "3 s-3 p laser gain and $\mathrm{x}$-ray line ratios for the carbon isoelectronic sequence," Journal of Applied Physics, 59(12), 3953-3957, 1986.

[18] U. Feldman, A.K. Bhatia, S. Suckewer, "Short wavelength laser calculations for electron pumping in neon-like krypton (Kr XXVII)," Journal of Applied Physics, 54(5), 2188-2197, 1983.

[19] U. Feldman, J.F. Seely, A.K. Bhatia, "Scaling of collisionally pumped 3 s-3 p lasers in the neon isoelectronic sequence," Journal of Applied Physics, 56(9), 2475-2478, 1984.
[20] W.H. Goldstein, J. Oreg, A. Zigler, A. Bar-Shalom, M. Klapisch, "Gain predictions for nickel-like gadolinium from a 181-level multiconfigurational distorted-wave collisional-radiative model," Physical Review A, 38(4), 1797, 1988. doi: $10.1137 / 083627$

[21] A. V Vinogradov, V.N. Shlyaptsev, "Calculations of population inversion due to transitions in multiply charged neon-like ions in the 200-2000 range," Soviet Journal of Quantum Electronics, 10(6), 754, 1980.

[22] I.I.S. Man, Introduction to the theory of atomic spectra, International series of Monographs in Natural Philosophy, 40, Pergamon Press.

[23] [FAC Code. http://kipac-tree.stanford.edu/fac].

[24] NIST [http://F:/NIST/NIST\%20ASD\%20Levels\%20Output32.htm].

[25] R.D. Neidinger, "Introduction to automatic differentiation and MATLAB object-oriented programming," SIAM Review, 52(3), 545-563, 2010. $10.1137 / 080743627$ 